\title{
Preface
}

\section{Advances in Trauma-2016}

\section{Goal Zero Preventable Deaths After Injury}

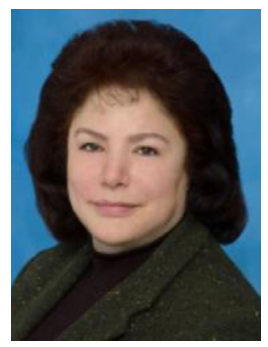

Lena M. Napolitano, MD, FACS, FCCP, MCCM Editor

Trauma remains the leading cause of death for individuals aged 1 to 46 years, the third leading cause of death overall, and accounts for $30 \%$ of all life-years lost, with over 190,000 lives lost annually. ${ }^{1}$ Trauma accounts for 41 million emergency department visits and over 2 million hospital admissions annually. The economic burden of trauma is more than $\$ 671$ billion annually. ${ }^{2}$ Despite an organized system of trauma care in the United States, from 2000 to 2010 , the number of trauma deaths increased by $22.8 \%$ for those 25 years and older with a concurrent increase in the US population of $9.7 \%$. The largest increase in trauma deaths was in 50- to 60-year-old individuals. ${ }^{3}$

So how can we provide optimal care to our trauma victims to ensure survival and optimal quality of life?

Standardization of evidence-based clinical care in trauma is of vital importance with a goal to improve trauma outcomes. Studies have documented low compliance rates (10\%-40\%) with standardized protocols in trauma. It has been confirmed that major deviations from guideline-based clinical care are associated with significantly higher mortality after injury and increased organ failure. ${ }^{4,5}$ Although we have standardized trauma care in the initial hour after injury with use of Advanced Trauma Life Support, there is little standardization subsequently. ${ }^{6}$

A new report from the National Academies of Sciences, Engineering, and Medicine (NASEM) proposes a national trauma care system integrating military and civilian trauma systems to achieve zero preventable deaths after injury. ${ }^{7}$ Recommendations encourage a culture of continuous learning and improvement, examination of unwarranted variation in practice, which has led to suboptimal patient outcomes, coordinated performance improvement and research to generate evidence-based best trauma care practices, structured quality improvement processes, and timely dissemination of trauma knowledge. ${ }^{8}$ The American College of Surgeons Committee on 
Trauma and the Coalition for National Trauma Research fully supports NASEM's report and recommendations. ${ }^{9,10}$ We now have to work on implementation of optimal care to improve trauma outcomes.

This issue of Critical Care Clinics provides an overview of the significant advances that have been made in trauma care to improve outcomes. Since the last update on trauma in Critical Care Clinics in 2004, a lot has changed.

In this issue, leading experts provide succinct reviews of important areas in trauma resuscitation and clinical care. They review cutting edge advances and provide evidence-based recommendations for best practices in trauma care. They include references to the American College of Surgeons Trauma Quality Improvement Program Best Practice Guidelines ${ }^{11}$ and provide personal insights to the care of complex and critically ill trauma victims.

I extend my deepest gratitude to our authors for their excellent contributions to this important issue on Advances in Trauma, and to Richard W. Carlson, Patrick J. Manley, and Casey Potter and the entire editorial team.

We hope that this issue of Critical Care Clinics will assist in timely dissemination of trauma knowledge by providing the updated evidence base to ensure optimal care to all of our trauma patients.

We have one ultimate goal: to achieve zero preventable deaths after injury.

Lena M. Napolitano, MD, FACS, FCCP, MCCM Division of Acute Care Surgery [Trauma, Burns, Surgical Critical Care, Emergency Surgery] Department of Surgery University of Michigan Health System Room 1C340A University Hospital 1500 East Medical Center Drive Ann Arbor, MI 48109-5033, USA

E-mail address: lenan@umich.edu

\section{REFERENCES}

1. Centers for Disease Control and Prevention, National Center for Injury Prevention and Control. Life years lost: a measure to account for the age at which deaths occur, giving greater weight to deaths occurring at younger ages and lower weight to deaths occurring at older ages. The LYL (percentage of total) indicator measures the LYL due to a particular cause of death as a proportion of the total LYL lost due to premature mortality in the population. Centers for Disease Control and Prevention. Web-based Injury Statistics Query and Reporting System (WISQARS). Available at: http://www.cdc.gov/injury/wisqars/. Accessed September 8, 2016.

2. Centers for Disease Control and Prevention, National Center for Injury Prevention and Control. Web-based Injury Statistics Query and Reporting System (WISQARS) 2015. Available at: http://www.cdc.gov/injury/wisqars. Accessed September 8, 2016.

3. Rhee P, Joseph B, Pandit V, et al. Increasing trauma deaths in the United States. Ann Surg 2014;260(1):13-21.

4. Rice TW, Morris S, Tortella BJ, et al. Deviations from evidence-based clinical management guidelines increase mortality in critically injured trauma patients. Crit Care Med 2012;40:778-86. 
5. Cuschieri J, Johnson JL, Sperry J, et al. Inflammation and Host Response to Injury, Large Scale Collaborative Research Program. Benchmarking outcomes in the critically injured trauma patient and the effect of implementing standard operating procedures. Ann Surg 2012;255(5):993-9.

6. Napolitano LM. Guideline compliance in trauma: evidence-based protocols to improve trauma outcomes? Crit Care Med 2012;40(3):990-2.

7. National Academies of Sciences, Engineering, and Medicine. A national trauma care system: integrating military and civilian trauma systems to achieve zero preventable deaths after injury. Washington, DC: National Academies Press; 2016.

8. Berwick DM, Downey AS, Cornett EA. A national trauma care system to achieve zero preventable deaths after injury: recommendations from a national academies of sciences, engineering, and medicine report. JAMA 2016;316(9):927-8.

9. Jenkins DH, Winchell RJ, Coimbra R, et al, American College of Surgeons Committee on Trauma. Position statement of the American College of Surgeons Committee on Trauma on the National Academies of Sciences, Engineering, and Medicine report. A national trauma care system: integrating military and civilian trauma systems to achieve zero preventable deaths after injury. J Trauma Acute Care Surg 2016. [Epub ahead of print].

10. Jenkins DH, Cioffi WG, Cocanour CS, et al, Coalition for National Trauma Research (CNTR). Position statement of the Coalition for National Trauma Research (CNTR) on the National Academies of Sciences, Engineering, and Medicine (NASEM) report, a national trauma care system: integrating military and civilian trauma systems to achieve zero preventable deaths after injury. J Trauma Acute Care Surg 2016. [Epub ahead of print].

11. ACS TQIP Best Practice Guidelines. Available at: https://www.facs.org/qualityprograms/trauma/taip/best-practice. 